\title{
Room temperature Bloch surface wave polaritons
}

\author{
Giovanni Lerario, ${ }^{1,5}$ Alessandro Cannavale, ${ }^{1,2}$ Dario Ballarini, ${ }^{2}$ Lorenzo Dominici, ${ }^{1,2}$ Milena De Giorgi, ${ }^{2}$ \\ Marco Liscidini, ${ }^{3}$ Dario Gerace, ${ }^{3}$ Daniele Sanvitto, ${ }^{2,6}$ and Giuseppe Gigli ${ }^{1,2,3}$ \\ ${ }^{1}$ CBN-IIT, Istituto Italiano di Tecnologia, Via Barsanti, 73010 Lecce, Italy \\ ${ }^{2} N N L$, Istituto Nanoscienze-Cnr, Via Arnesano, 73100 Lecce, Italy \\ ${ }^{3}$ Dipartimento di Fisica, Università di Pavia, via Bassi 6, I-27100 Pavia, Italy \\ ${ }^{4}$ University of Salento, Via Arnesano, 73100 Lecce, Italy \\ ${ }^{5}$ e-mail: giovannilerario@hotmail.com \\ ${ }^{6}$ e-mail: daniele.sanvitto@nano.cnr.it
}

Received January 20, 2014; accepted February 18, 2014;

posted February 27, 2014 (Doc. ID 204924); published March 27, 2014

Polaritons are hybrid light-matter quasi-particles that have gathered a significant attention for their capability of showing room temperature and out-of-equilibrium Bose-Einstein condensation. More recently, a novel class of ultrafast optical devices have been realized by using flows of polariton fluids, such as switches, interferometers, and logical gates. However, polariton lifetimes and propagation distances are strongly limited by photon losses and accessible in-plane momenta in normal microcavity samples. In this work, we show experimental evidence of the formation of room temperature propagating polariton states arising from the strong coupling between organic excitons and a Bloch surface wave. This result, which was only recently predicted, paves the way for the realization of polariton devices that could allow lossless propagation up to macroscopic distances. ( 2014 Optical Society of America

OCIS codes: (240.6690) Surface waves; (240.5420) Polaritons; (160.4890) Organic materials; (230.1480) Bragg reflectors; (240.0310) Thin films.

http://dx.doi.org/10.1364/OL.39.002068

Mixed light-matter excitations, also called polaritons, arise from the nonperturbative coupling of optical transitions with large oscillator strength and electromagnetic modes propagating or confined in semiconducting or insulating media. [1,ㄹ] Recently, polariton excitations in 2D geometries have been attracting considerable interest, both for fundamental studies on out-of-equilibrium Bose-Einstein condensation and superfluidity, [3-7] and for the possibility of exploiting their nonlinear properties for all-optical operations. [,ㅜㄹ] Elementary excitations can be observed in materials such as squaraine, porphyrin, and cyanine dyes. As opposed to Wanniertype exciton polaritons, their ultrahigh oscillator strength allows for the observation of polariton effects at room temperature and for Rabi splittings that could reach energies on the order of the excitonic transition.

So far, most of the research on polaritons concerning both Wannier and organic types $[10,11]$ has been mainly focused on the strong coupling to the photonic modes of high-finesse planar microcavities, where the active medium is embedded in a cavity layer between two high-reflectivity mirrors. However, planar microcavities offer little freedom in engineering the propagation of polaritons, due to their small accessible momenta. Recently, it has been suggested that similar phenomena could also be observed at the interface between a single distributed Bragg reflector (DBR) and a homogeneous medium by exploiting strong light-matter coupling to Bloch surface waves (BSWs). [12,13] Such Bloch surface wave polaritons (BSWP), [14] i.e., mixed excitations bound to the surface of the Bragg mirror, which exploit the confinement of the optical mode due to total internal reflection as well as a photonic bandgap, could be exploited for prospective polariton devices with high efficiency and controlled long range propagation or for applications requiring high surface sensitivity such as optical sensors, $[\underline{15}, \underline{16}]$ with clear advantages over alternative structures where metal deposition is required. $[17,18]$ Furthermore, this is a promising system for the study of nonlinear optical effects $[19,20]$ due to the excitonic counterpart; different from other lossless surface

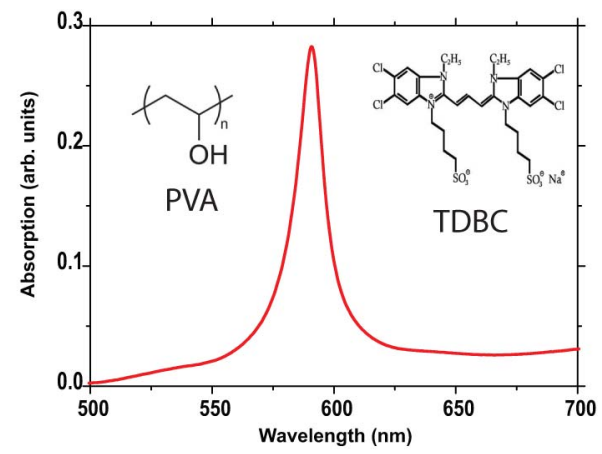

(a)

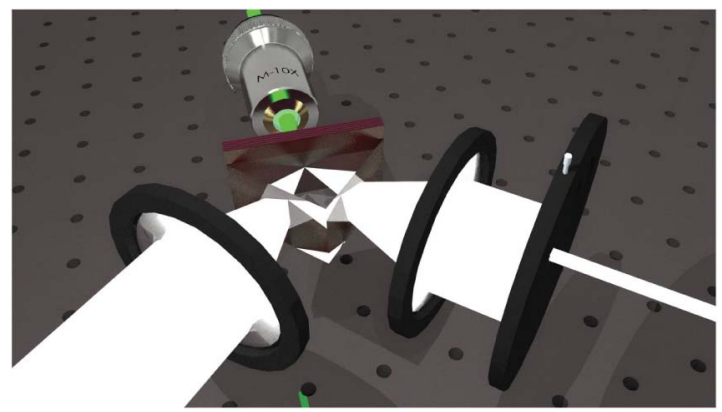

(b)

Fig. 1. (a) Absorption and chemical formula of the TDBC molecule in the J-aggregate form. (b) Schematic drawing of the experimental setup used for reflectivity and emission measurements. 
waves generated between linear media, such as optical Tamm states or Dyakonov surface states.

In this Letter, we report on the experimental demonstration of strong light-matter coupling between J-aggregate excitons with BSWs at the interface between $\mathrm{a} \mathrm{SiO}_{2} / \mathrm{TiO}_{2}$ Bragg mirror and an index-matching homogeneous medium. Evidence of polariton formation is reported by independently measuring reflectivity and emission spectra, which both show the typical anticrossing behavior as a function of the angle of incidence.

The sample is made of five pairs of $\lambda / 4 \mathrm{TiO}_{2} / \mathrm{SiO}_{2}$ $(101 \mathrm{~nm} / 118 \mathrm{~nm})$ alternating layers deposited on a glass substrate ending with an additional couple of $\mathrm{TiO}_{2} / \mathrm{SiO}_{2}$ $(14 \mathrm{~nm} / 16 \mathrm{~nm})$ layers that allow for an optimal tuning of the BSW energy position as well as its field enhancement at the surface of the DBR. On top of the mirror we have spin coated a layer of polyvinyl alcohol (PVA) (Sigma Aldrich) and 5,6-Dichloro-2-[[5,6-dichloro-1-ethyl-3-(4sulfobutyl)-benzimidazol-2-ylidene]-propenyl]-1-ethyl-3(4-sulfobutyl)-benzimidazolium hydroxide, inner salt, sodium salt (TDBC) (FEW Chemicals). The latter is a thoroughly studied cyanine dye which shows a marked property of J-aggregation both in solution and in solid state. [21] Such a characteristic results in a narrow absorption peak around $590 \mathrm{~nm}$ with a relatively thin bandwidth compared to its monomer absorption. The PVA matrix is a polymer with a large energy bandgap (about $5.3 \mathrm{eV}$ ), which is not affecting the optical properties of the TDBC. The deposition of this last layer is obtained starting from a solution of PVA:TDBC (1:1 weight ratio) dissolved in a mixture of methanol:water (1:1 volume ratio). The concentration of both materials in the final solution is of $0.5 \mathrm{mg} / \mathrm{ml}$ and additional stirring, ultrasonic bath and heating treatments are adopted in the solution. The final layer thickness is about $20 \mathrm{~nm}$; we spin coated an ultrathin layer in order to retain good visibility of the modes of the system, which could be reduced due to the uncoupled molecule absorption.

Figure 1(a) shows the molecular structure of PVA and TDBC and the absorption spectrum of the PVA:TDBC composite layer deposited on a glass substrate. From the absorption spectrum the complete formation of J-aggregates is deducible, with a full width at half-maximum (FWHM) of about $45 \mathrm{meV}$ and the absence of any peaks ascribed to the TDBC monomers (usually positioned at $520 \mathrm{~nm}$ ). Reflectivity measurements have been done using a goniometric system with a white light source focused on the sample surface and collected at the output via two high numerical aperture lenses. Detection of different reflection angles is done by selection of the light at the Fourier plane as shown by the sketch in Fig. 1(b). The light is finally focalized on a monochromator and the spectra are revealed by a charged coupled device (CCD) camera. This setup also allows us to obtain an imaging of the sample surface so that a specific area of interest can be selected. The overall angular resolution is about $0.2^{\circ}$. The measurements are obtained in the Kretschmann configuration by means of an index-matching oil. Photoluminescence, instead, is carried out by a $532 \mathrm{~nm} \mathrm{cw}$ laser exciting the PVA:TDBC from the top side of the sample [see Fig. 1(b)].

Reflection and emission spectra are shown in Fig. 2 for different angles of incidence relative to the sample normal in the prism. The vertical dotted line indicates the absorption peak energy position of the bare TDBC aggregates. The reflectance dip (emission peak) is due to the excitation of a BSW at the sample surface. As can be seen by tuning the BSW across the TDBC absorption, the mode splitting into the upper and lower polariton branches is clearly visible in both the reflectivity as well
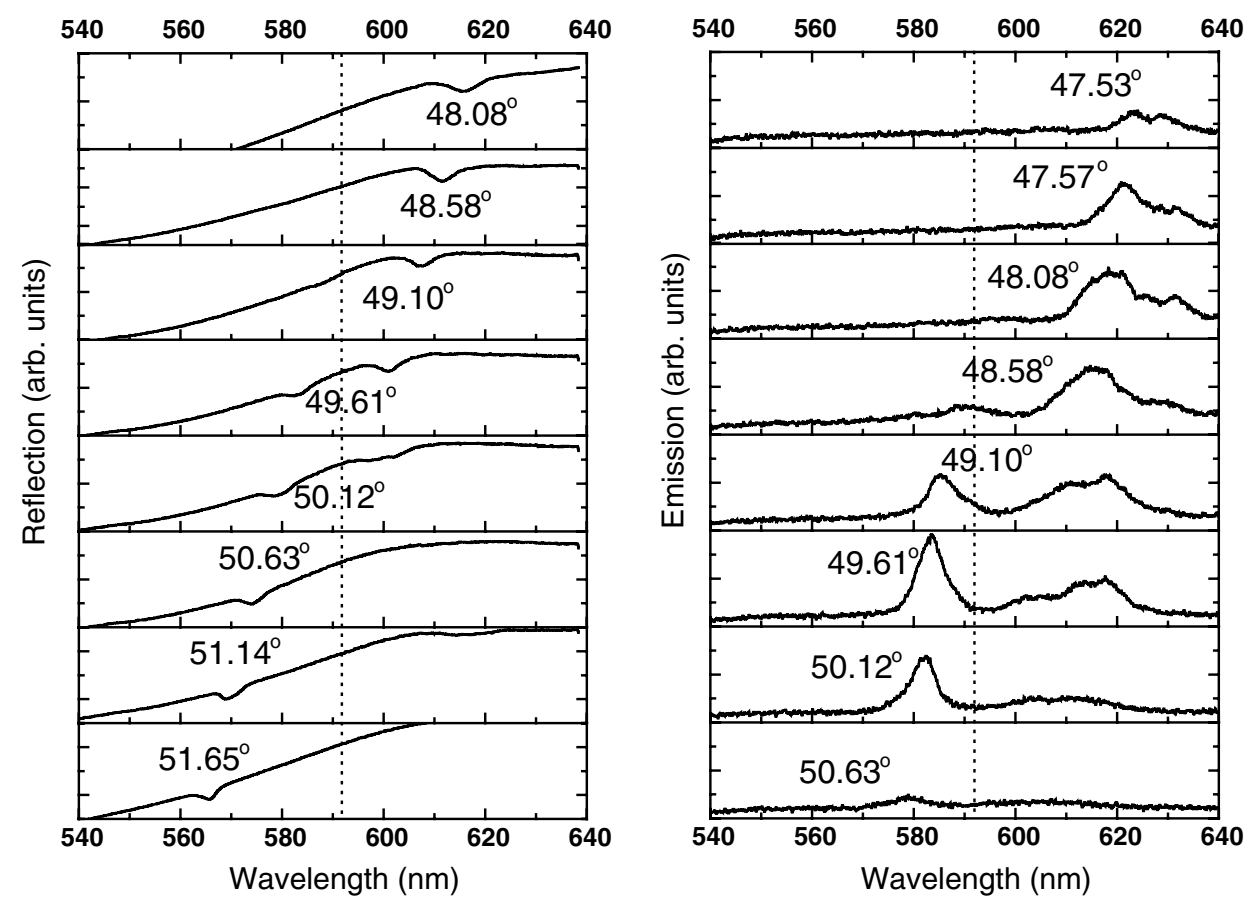

Fig. 2. Reflectivity (left) and photoluminescence (right) spectra taken at different angles of incidence with respect to the normal to the sample inside prism. 
(a)

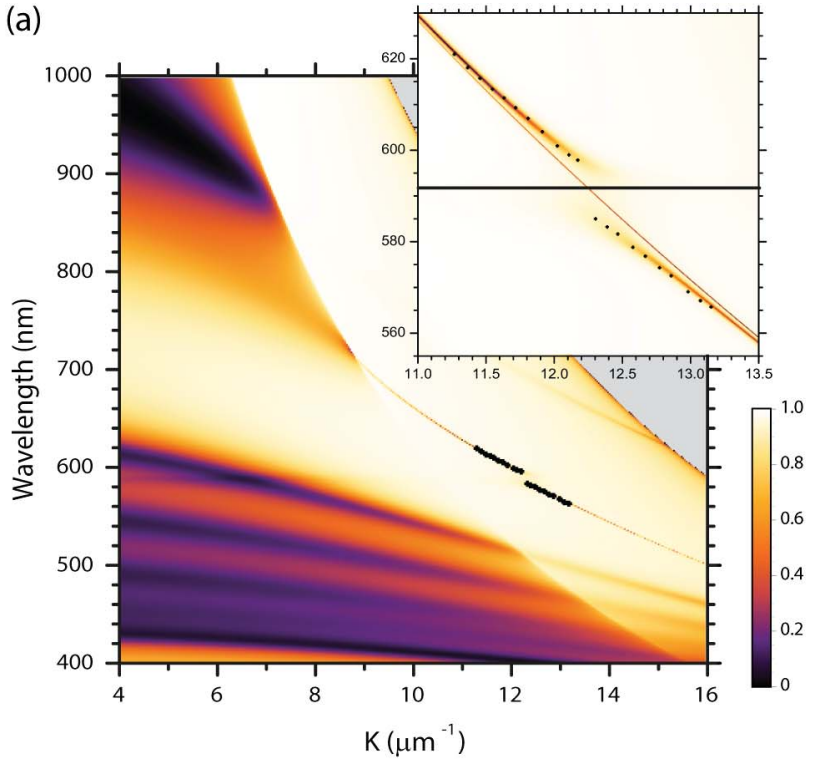

(b)

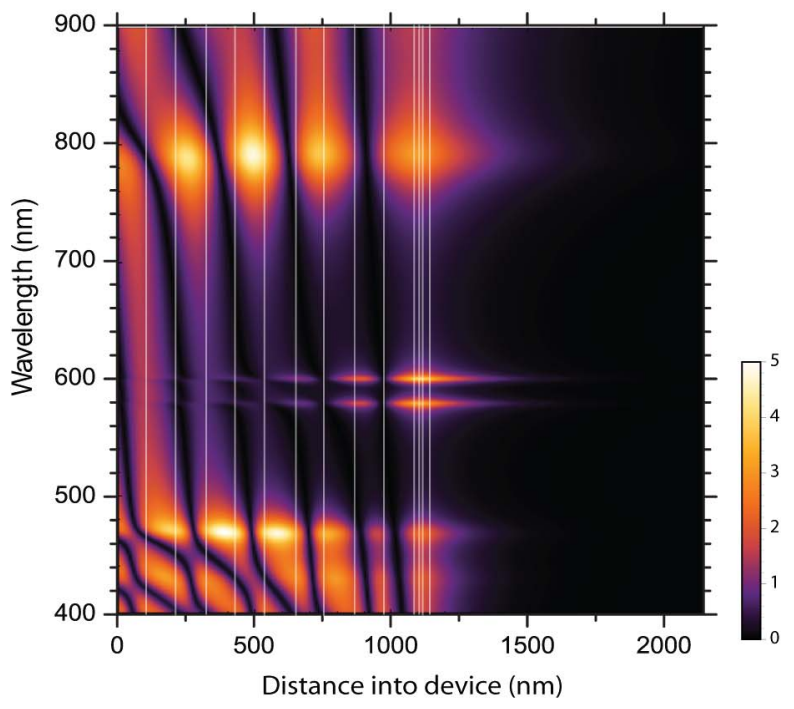

Fig. 3. (a) Experimental data and theoretical calculation of the reflectivity as a function of wavelength and angle of incidence. The light line corresponds to an angle of about $42 \mathrm{deg}$; here, the Bloch surface mode starts to appear from the edge of the Bragg mirror. At $k=12-12.5 \mu \mathrm{m}^{-1}$ the anticrossing with the exciton of the TDBC is visible, as evidenced in the expanded inset (horizontal line is the exciton, diagonal line is the unmodified BSW mode dispersion). (b) Calculated enhancement of the electromagnetic field at 50.54 deg as a function of wavelength and distance from the bottom of the Bragg reflector.

as the emission spectra, showing the typical anticrossing signature of the strong coupling regime with a Rabi splitting of about $45 \mathrm{meV}$.

At the anticrossing point we can estimate a polariton FWHM of $14 \mathrm{meV}$, while the bare BSW linewidth is below the measurement resolution of $10 \mathrm{meV}$. This is caused by the very strong variation of the mode energy with angle, while the selectivity is limited by the total detection efficiency and lens aberrations.

Theoretical calculations of the reflectivity spectra and the field distribution using the transfer matrix method [12] are shown in Fig. 3.

All the DBR layer parameters have been extracted from ellipsometric measurements on the deposited materials, with only very small adjustments accounting for thickness variation during the deposition process. We have simulated the TDBC aggregates by a single Lorentz oscillator that fits the experimental absorption line and refractive indexes; [21,22] the effective refractive index, dependent on the cross section ratio between the two materials, is then optimized in order to match the layer thickness and the Rabi splitting deduced from Fig. 2.

In Fig. 3(a) the experimental values of the reflectivity spectra in wavelength-momentum space have been plotted together with the simulated data of the sample reflectance. in this case including also the region within the lightcone in air. As can be seen, by increasing the momentum, the DBR stop band (visible as a bright region) shifts toward higher energy.

Once the total internal reflection angle is reached (at about 42 deg in glass) the BSW mode starts to appear at the edge of the mirror stop band, showing a much more abrupt dispersion. When the mode reaches the energy of the TDBC absorption peak (horizontal black line in the inset) the splitting due to the strong coupling between the BSW mode and the exciton of the dye starts to appear in perfect accordance with the experimental data of Fig. 2. The coupling appears as a deviation from the purely optical BSW [continuous diagonal line in the inset in Fig. 3(a)].

Within the theoretical calculations we obtain a polariton FWHM band of $14 \mathrm{meV}$, in agreement with the experimental value, while the BSW bandwidth is only $5 \mathrm{meV}$, which suggests that the $10 \mathrm{meV}$ obtained experimentally is effectively given by limitations in the overall setup resolution.

Finally, in Fig. 3(b) we have plotted the field enhancement of the BSW as a function of wavelength and distance from the sample surface, taken at an angle of incidence of $50.45 \mathrm{deg}$. The vertical white lines indicate the order of the layers composing the DBR mirror. The final phase matching layers of the DBR concentrate the highest intensity of the electric field of the surface mode and, as can be seen in Fig. 3(b), allow for the best coupling into the $20 \mathrm{~nm}$ thick PVA:TDBC final layer.

In conclusion, we have experimentally demonstrated the room temperature formation of Bloch surface wave polaritons at the interface between a Bragg mirror and a homogeneous medium. Such quasi-particles arise from the strong coupling between the optical transition of an organic J-aggregate compound deposited on top of the Bragg mirror surface, and the Bloch surface mode existing at the interface. Given the increasing interest in organic polaritons and the potentialities of surface waves for sensing, these results will stimulate further research on strongly coupling $2 \mathrm{D}$ layered materials to suitably engineered surface photonic modes and long lasting polariton waves.

G. L. is grateful to Gianluca Latini for the encouragement at the initial stage of his research path. This work has been funded by the MIUR project Beyond Nano and the ERC project POLAFLOW (Grant 308136). 


\section{References}

1. L. C. Andreani, in Electron and Photon Confinement in Semiconductor Nanostructures, B. Deveaud, A. Quattropani, and P. Schwendimann, eds. (IOS, 2003), p. 105.

2. For a recent review: A. V. Kavokin, Phys. Status Solidi B 247, 1898 (2010).

3. J. Kasprzak, M. Richard, S. Kundermann, A. Baas, P. Jeambrun, J. M. J. Keeling, F. M. Marchetti, M. H. Szymaska, R. André, J. L. Staehli, V. Savona, P. B. Littlewood, B. Deveaud, and L. S. Dang, Nature 443, 409 (2006).

4. R. Balili, V. Hartwell, D. Snoke, L. Pfeiffer, and K. West, Science 316, 1007 (2007).

5. A. Amo, D. Sanvitto, F. P. Laussy, D. Ballarini, E. del Valle, M. D. Martin, A. Lemaître, J. Bloch, D. N. Krizhanovskii, M. S. Skolnick, C. Tejedor, and L. Viña, Nature 457, 291 (2009).

6. A. Amo, J. Lefrère, S. Pigeon, C. Adrados, C. Ciuti, I. Carusotto, R. Houdré, E. Giacobino, and A. Bramati, Nat. Phys. 5, 805 (2009).

7. I. Carusotto and C. Ciuti, Rev. Mod. Phys. 85, 299 (2013).

8. T. C. H. Liew, A. V. Kavokin, and I. A. Shelykh, Phys. Rev. Lett. 101, 016402 (2008).

9. D. Ballarini, M. De Giorgi, E. Cancellieri, R. Houdré, E. Giacobino, R. Cingolani, A. Bramati, G. Gigli, and D. Sanvitto, Nat. Commun. 4, 1778 (2013).

10. M. S. Skolnick, T. A. Fisher, and D. M. Whittaker, Semicond. Sci. Technol. 13, 645 (1998).
11. V. M. Agranovich and G. C. La Rocca, Solid State Commun. 135, 544 (2005).

12. A. Yariv and P. Yeh, Optical Waves in Crystals (Wiley, 2003).

13. E. Descrovi, F. Giorgis, L. Dominici, and F. Michelotti, Opt. Lett. 33, 243 (2008).

14. M. Liscidini, D. Gerace, D. Sanvitto, and D. Bajoni, Appl. Phys. Lett. 98, 121118 (2011).

15. M. Liscidini and J. E. Sipe, J. Opt. Soc. Am. 26, 279 (2009).

16. E. Descrovi, T. Sfez, M. Quaglio, D. Brunazzo, L. Dominici, F. Michelotti, H. P. Herzig, O. J. F. Martin, and F. Giorgis, Nano Lett. 10, 2087 (2010).

17. M. Kaliteevski, S. Brand, R. A. Abram, I. Iorsh, A. V. Kavokin, and I. A. Shelykh, Appl. Phys. Lett. 95, 251108 (2009).

18. C. Symonds, A. Lemaître, E. Homeyer, J. C. Plenet, and J. Bellessa, Appl. Phys. Lett. 95, 151114 (2009).

19. K. S. Daskalakis, S. A. Maier, R. Murray, and S. Kéna-Cohen, Nat. Mater. 13, 271 (2014).

20. J. D. Plumhof, T. Stöferle, L. Mai, U. Scherf, and R. F. Mahrt, Nat. Mater. 13, 247 (2014).

21. J. R. Tischler, M. S. Bradley, and V. Bulovic, Opt. Lett. 31, 2045 (2006).

22. C. Neipp, S. Gallego, M. Ortuño, A. Marrquez, A. Beléndez, and I. Pascual, Opt. Commun. 224, 27 (2003). 